\title{
SCIENTIFIC REPORTS

\section{OPEN Role of Clathrin Assembly Protein-2 Beta Subunit during White Spot Syndrome Virus Infection in Black Tiger Shrimp Penaeus monodon}

Received: 5 June 2019
Accepted: 2 September 2019 Published online: 17 September 2019

\author{
Thapanan Jatuyosporn ${ }^{1,2}$, Pasunee Laohawutthichai ${ }^{1,2}$, Premruethai Supungul ${ }^{3}$, \\ Rogerio R. Sotelo-Mundo $\mathbb{D}^{4}{ }^{4}$, Adrian Ochoa-Leyva ${ }^{5}$, Anchalee Tassanakajon ${ }^{2}$ \& \\ Kuakarun Krusong ${ }^{1}{ }^{1}$
}

White spot syndrome virus (WSSV) is one of the most lethal viruses severely affecting shrimp industry. This disease can cause $100 \%$ mortality of farmed shrimp within a week. This work aims to characterize clathrin assembly proteins in Penaeus monodon and investigate their roles in WSSV entry. In general, clathrin assembly proteins form complexes with specific receptors and clathrins, leading to clathrinmediated endocytosis. Adaptor protein 2 (AP-2), which is responsible for endocytosis at plasma membrane, consists of four subunits including $\alpha, \beta 2, \mu 2$ and $\sigma 2$. Knockdown of clathrin coat AP17, or $\sigma$ subunit of AP-2 dramatically reduced WSSV infectivity. Similar results were observed, when shrimp were pre-treated with chlorpromazine (CPZ), an inhibitor of clathrin-dependent endocytosis. The complete open reading frames of AP- $2 \beta$ and $\mu$ subunits of $P$. monodon are reported. PmAP-2 $\beta$ was up-regulated about 4-fold at 6 and $36 \mathrm{~h}$ post-WSSV infection. Knockdown of PmAP-2 $\beta$ delayed shrimp mortality during WSSV infection, of which WSSV intermediate early 1 gene expression was also down-regulated. Immunogold-labelling and transmission electron microscopy revealed that PmAP-2 $\beta$ co-localized with WSSV particles at plasma membrane. In addition, PmAP-2 $\beta$-silencing significantly affected the expression levels of PmSTAT, PmDOME, PmDorsal and ALFPm3 during WSSV infection. It is possible that PmAP-2 $\beta$ is associated with the JAK/STAT and the Toll pathway.

White spot syndrome virus (WSSV) is a highly pathogenic virus that causes massive death in penaeid shrimp. Once infected by WSSV, 100\% cumulative mortality of farmed shrimps could be expected within 3-10 days ${ }^{1}$. WSSV is a non-occluded, enveloped, rod-shaped, double stranded DNA virus, which belongs to the genus Whispovirus and family Nimaviridae ${ }^{2,3}$. The rod-shaped, intact virus particle is about $70-167 \mathrm{~nm} \times 210-380 \mathrm{~nm}^{4-8}$ and contains the genome of approximately $300 \mathrm{kbp}$, depending on the viral isolate ${ }^{9-11}$. Several reports have suggested clathrin-mediated endocytosis involves in WSSV infection in the pacific white shrimp Litopenaeus vannamei $^{12}$ and the red claw crayfish Cherax quadricarinatus hematopoietic tissue cells ${ }^{13,14}$. Both DNA viruses, such as African swine fever virus, Vaccinia virus and Singapore Grouper Iridovirus, and RNA viruses, including Ebolavirus, Hepatitis C virus, Influenza A virus, Dengue virus and Yellow head virus, are internalized via clathrin-dependent endocytosis ${ }^{15-22}$.

Clathrin-mediated endocytosis is a well-characterized process responsible for the transportation of a wide variety of molecules from the cell surface inside the cells. Clathrin-adaptor protein 2 (AP-2) is responsible for

${ }^{1}$ Structural and Computational Biology Research Unit, Department of Biochemistry, Faculty of Science, Chulalongkorn University, Bangkok, 10330, Thailand. ${ }^{2}$ Center of Excellence for Molecular Biology and Genomics of Shrimp, Department of Biochemistry, Faculty of Science, Chulalongkorn University, Bangkok, 10330, Thailand. ${ }^{3}$ National Center for Genetic Engineering and Biotechnology (BIOTEC), National Science and Technology Development Agency (NSTDA), Pathumthani, 12120, Thailand. ${ }^{4}$ Laboratorio de Estructura Biomolecular, Centro de Investigación en Alimentación y Desarrollo, A.C. (CIAD), Carretera Gustavo Enrique Astiazaran Rosas No. 46, Hermosillo, Sonora, 83304, Mexico. ${ }^{5}$ Departamentos de Microbiología Molecular, Medicina Moleculary Bioprocesos, Unidad Universitaria de Secuenciacián Masiva y Bioinformática, Instituto de Biotecnología (IBT), Universidad Nacional Autónoma de México (UNAM), Avenida Universidad 2001, Colonia Chamilpa, Cuernavaca, 62210, Mexico. Correspondence and requests for materials should be addressed to K.K. (email: Kuakarun.K@chula.ac.th) 


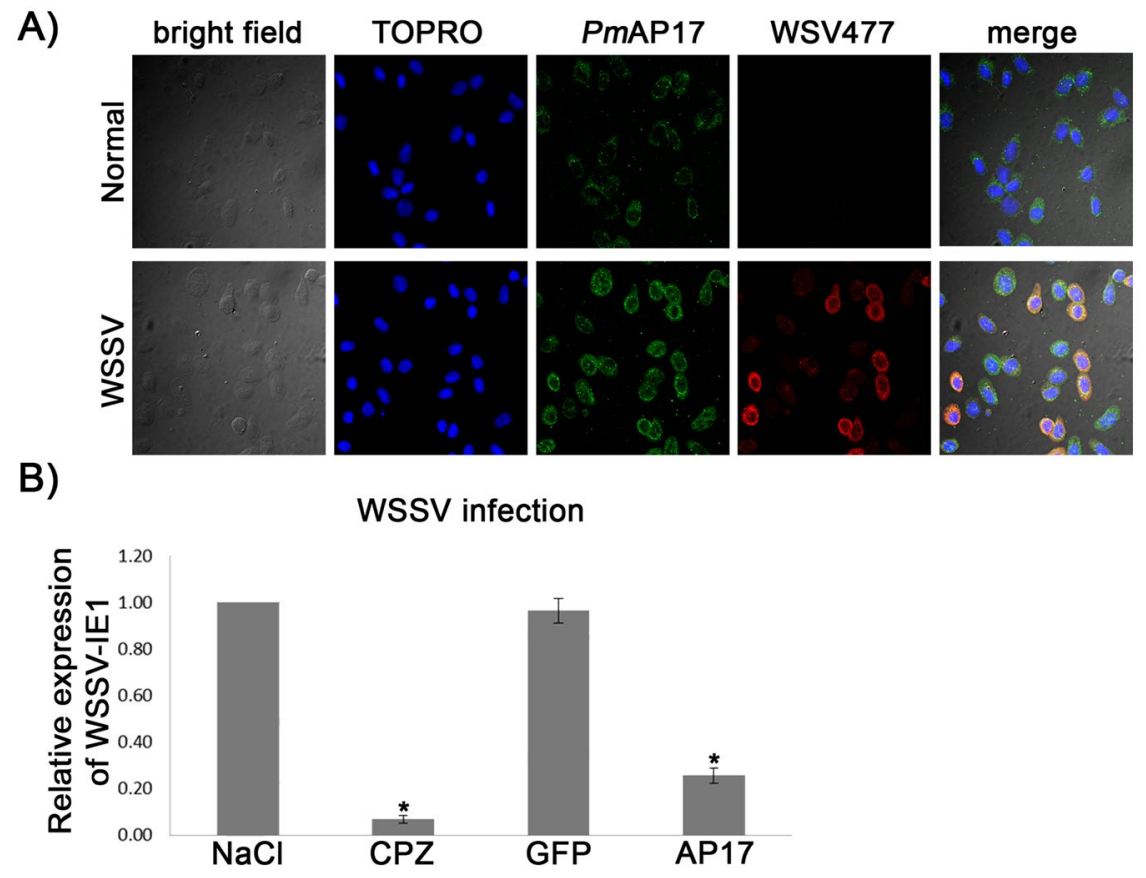

Figure 1. Effect of $P m$-clathrin coat AP17 silencing and chlorpromazine on WSSV propagation. (A) Immunofluorescent staining analysis of Pm-clathrin coat AP17 and WSV477 in hemocytes by confocal laser scanning microscopy. Hemocytes from unchallenged and WSSV challenged were collected at 24 hpi and probed by anti-Pm-clathrin coat AP17 and WSV477 antibodies. Pm-clathrin coat AP17 and WSV477 proteins were visualized by secondary antibodies conjugated with Alexa Fluor 488 (green) and Alexa Fluor 568 (red), respectively. Nuclei were stained in blue. (B) The evaluated replicativity of WSSV in Pm-clathrin coat AP17 silencing and chlorpromazine pre-treated shrimp. Shrimp were divided into four groups and injected with either $150 \mathrm{mM} \mathrm{NaCl}$, GFP dsRNA (10 $\mu \mathrm{g} / \mathrm{g}$ shrimp), Pm-clathrin coat AP17 dsRNA (10 $\mu \mathrm{g} / \mathrm{g}$ shrimp) or chlorpromazine $(0.25 \mu \mathrm{g} / \mathrm{g}$ shrimp). After WSSV infection, the hemocyte were collected and used for determination of WSSV replicativity. The mRNA expression levels of WSSV IE1 were analyzed by Quantitative Real-time RT-PCR. The data are shown as the mean \pm standard deviation. An asterisk represents significant differences from control group $(p<0.05)$. The experiment was carried out in triplicates.

endocytosis at the plasma membrane while AP-1 and AP-3 complexes participate in endocytic vesicle formation at the trans-Golgi network and at the membrane of lysosomes, respectively ${ }^{23}$. In general, AP- 2 complex consists of 4 subunits: 2 large subunits $(\alpha$ and $\beta$ ), one medium subunit $(\mu)$ and one small subunit $(\sigma)$. The $\alpha$ subunit recruits AP-2 complex to plasma membrane by interacting with phosphatidylinositol 4,5-bisphosphate $\left(\mathrm{PI}(4,5) \mathrm{P}_{2}\right)^{24}$, while the $\beta 2$ subunit links AP-2 with clathrin and may play a role in the selection of specific cargo ${ }^{25}$. The $\mu 2$ subunit recognizes and sorts protein cargo $^{26}$. The well-characterized sorting signals within transmembrane cargo molecules are tyrosine-based (YXXØ) and dileucine-based [DE]XXXL[LI] signals, when X is any amino acid residues, $\varnothing$ represents a bulky hydrophobic amino acid and the brackets indicates either amino acid is allowed at that position ${ }^{27}$. Tyrosine-based signals interact with AP2 complexes through the binding with $\mu 2$, while dileucine-based signals bind to $\alpha-\sigma 2$ subunits. Previously, the $\sigma$ subunit of AP-2, known as AP17 in P. monodon, has been characterized ${ }^{22}$.

This study reports, for the first time, two main components of AP-2 complexes in P. monodon, PmAP-2 $\beta$ and PmAP2- $\mu$, which play a part in cargo recognition. RNA interference technique, endocytosis inhibitor, immunofluorescence confocal microscopy and transmission electron microscopy (TEM) were used to investigate WSSV infection via clathrin-dependent endocytosis.

\section{Results}

Silencing of Pm-clathrin coat AP17 and chlorpromazine (CPZ) treatment reduced WSSV propagation. Immunofluorescence confocal microscopy showed that Pm-clathrin coat AP17 was found in the cytoplasm of shrimp hemocytes and the level of Pm-clathrin coat AP17 was higher in WSSV infected shrimp hemocytes, in comparison to non-infected shrimp (Fig. 1A). Pm-clathrin coat AP17 (green) was also localized with WSV477 (red) in the cytoplasm of shrimp hemocytes. Silencing of Pm-clathrin coat AP17 and CPZ pre-treatment resulted in a significant reduction of WSSV-IE1 transcripts at 12 hpi (Fig. 1B). Pm-clathrin coat AP17 gene knockdown lower WSSV-IE1 expression by 4.3-fold, while CPZ treatment reduced WSSV-IE1 transcription level by 12.5 -fold. Both suggested that clathrin-mediated endocytosis plays a role in WSSV entry.

Gene cloning, bioinformatics analysis and recombinant protein production of PmAP-2 $\beta$ and

PmAP-2 $\mu$. To further investigate role of clathrin-mediated endocytosis during WSSV infection, other clathrin 
adaptor proteins, PmAP-2 $\beta$ and PmAP- $2 \mu$ were studied. The complete 2,820 bp PmAP- $2 \beta$ cDNA sequence was obtained from the P. monodon EST database (http://pmonodon.biotec.or.th). The open reading frame (ORF) of PmAP-2 $\beta$ (GenBank accession number MK089559) can be translated into a protein of 939 amino acid residues (Fig. 2A), with a predicted molecular weight of $103 \mathrm{kDa}$ and $\mathrm{pI}$ of 4.98.

The deduced amino acid sequence of PmAP-2 $\beta$ showed high sequence similarity to the putative AP- 2 complex subunit $\beta$-1 from Litopenaeus vannamei (ROT82409.1, 98\% identity), followed by PmAP- $2 \beta$ from Ixodes species (acc. NO.GEFM01002526.1 and XP_002412040.1, 81.1\% identity) and from Anopheles species (acc. NO. A0A182FRP2 and GGFK01000869.1, 78.2\% identity). The phylogenetic trees of AP-2 complex subunit $\beta$ from vertebrates and invertebrates are displayed in Fig. 2B. Multiple sequence alignment suggests amino acid differences in 60 positions that distinguish AP- 2 complex subunit $\beta$ in vertebrates from those in invertebrates (Fig. 3 ).

Previously, the crystal structure of Homo sapiens AP- $2 \beta$ revealed that the protein possesses three domains, including the $\mathrm{N}$-terminal trunk domain, the $\mathrm{C}$-terminal appendage domain and the flexible hinge region located between $\mathrm{N}$ - and $\mathrm{C}$-terminal domains ${ }^{28}$. The $\mathrm{N}$-terminal domain of PmAP- $2 \beta$ shares strong sequence identity (82.53\%) with HmAP-2 $3^{28}$, however, the C-terminal domain has less sequence identity but shows similar structural fold ${ }^{29}$. In Homo sapiens, C-terminal appendages bind with [DE $]_{n} \mathrm{X}_{1-2} \mathrm{FXX}[\mathrm{FL}] \mathrm{XXXR}$ motif in clathrin-coated accessory proteins such as Epsin 1, Epsin 2, $\beta$-arrestin 1, $\beta$-arrestin 2 and autosomal recessive hypercholesterolemia (ARH) via R834, F837, L838, W841, E849, A877, R879, Y888 and E902. These amino acid residues are highly conserved in invertebrates, but Arginine at position 834 (according to HmAP-2 $\beta$ ) is replaced by Lysine residue in invertebrates. The flexible hinge region has been reported to interact with N-terminal of clathrin heavy chain using a canonical clathrin box motif (LLNLD) ${ }^{30,31}$, which is strongly conserved in vertebrates (Fig. 3). However, this motif in invertebrates is less conserved. It presents as LLSMD in insecta (Ixodes Ricinus, Ixodes scapularis, Anopheles albimanus and Anopheles triannulatus) and LLNMD in P. monodon.

PmAP-2 $\beta$ was expressed in Escherichia coli BL21 CodonPlus and the recombinant protein was purified by $\mathrm{Ni}$ Sepharose 6 Fast Flow under denaturing condition and then refolded. In Fig. S1 in Supplementary Information, PmAP-2 $\beta$ showed an apparent molecular weight of approximately $110 \mathrm{kDa}$, which is closed to the calculated molecular weight of His-tagged fusion PmAP-2 $\beta$.

In addition, 5' - Rapid Amplification of cDNA Ends (5'-RACE) was performed to obtain the full-length PmAP-2 $\mu$ cDNA sequence of 1,299-bp (GenBank accession number MK496174), which encodes 432 amino acids. PmAP- $2 \mu$ has calculated molecular weight of $49.3 \mathrm{kDa}$ and $\mathrm{pI}$ of 9.55. In Fig. S1 in Supplementary Information, the recombinant His-tagged fusion PmAP2 $\mu$ was successfully purified as a single band with an apparent molecular weight of $53 \mathrm{kDa}$. Based on protein sequence analysis, PmAP- $2 \mu$ contains two major domains, including longin-like domain at the $\mathrm{N}$-terminus and mu homology domain at the C-terminus. As shown in Fig. S2 in Supplementary Information, the conserved longin-like domain consists of $\beta 1, \beta 2, \mathrm{H} 1, \beta 2, \beta 4, \beta 5, \mathrm{H} 2$ and $\mathrm{H} 3$, which interacts with AP- $2 \beta$ subunit to form clathrin assembly protein complexes ${ }^{28}$, while the mu homology domain has all $\beta$-sheet structure ( $\beta 6-\beta 23)$.

According to protein BLAST analysis and sequence homology search (BlastX), PmAP2 $\mu$ revealed high sequence similarity to the AP-2 complex subunit $\mu$ from Litopenaeus vannamei (XP_027231007.1, 99\% identity), clathrin-associated adaptor protein complexes $\mu$ subunit from C. quadricarinatus (ALP46597.1, 98\% identity), as well as AP-2 complex subunit $\mu$ from Agrilus planipennis, Onthophagus taurus, Tribolium castaneum, Diabrotica virgifera virgifera and Anoplophora glabripennis (XP_018335920.1, XP_022918165.1, NP_001280510.1, ATD50466.1 and XP_018578400.1, respectively, 91\% identity). The phylogenetic trees revealed that AP-2 complex subunit $\mu$ can be divided into two main groups: vertebrates and invertebrates, and each of them contains two subgroups (Fig. S2). In invertebrates, AP- $2 \mu$ is classified into Arthropoda subgroup (P. monodon, L. vannamei and C. quadricarinatus) and insect subgroup (Nicrophorus vespilloides, Zootermopsis nevadensis and Bicyclus anynana). In vertebrates, AP- $2 \mu$ sequences from Homo sapiens, Bos taurus and Mus musculus are identical. Based on multiple sequence alignments, vertebrate and invertebrate AP- $2 \mu$ can be distinguished by the amino acid residues at 14 different locations: two positions are on longin-like domain, while the other twelve locate on mu homology domain (Fig. S3 in Supplementary Information). T156 of Homo sapiens AP-2 $\mu$ has been reported to be phosphorylated and essential for interacting with transferrin receptors in vitro and in vivo ${ }^{32,33}$, as well as for binding affinity of AP-2 complex to YXX $\phi$ motif of the cargo proteins ${ }^{34,35}$. This threonine corresponds to T154 in P. monodon and is highly conserved in all organisms. Moreover, seven crucial amino acid residues for vesicle traffic that interact to phosphoinositide lipids ${ }^{25,28}$, are also highly conserved across diverse phyta. These amino acid residues of Homo sapiens AP-2 $\mu$ are K319, E321, K341, K343, K345, K354 and K356, corresponding to K316, E318, K338, K340, K342, K351 and K353 in PmAP-2 $\mu$ as shown in Fig. S3.

Analysis of PmAP-2 $\beta$ gene expression. Ten tissues (hemocyte, eyestalk, epipodite, gill, heart, lymphoid organ, stomach, intestine, hepatopancreas and muscle) were collected to determine PmAP- $2 \beta$ expression. The PCR products were analyzed by $1.5 \%$ agarose electrophoresis and measured intensity of PmAP- $2 \beta$ and EF- $1 \alpha$ (an internal control) by Gel Analyzer software. Among the tissues, PmAP-2 $\beta$ was expressed at the highest level in hemocyte and gill, while the lowest amount of PmAP- $2 \beta$ transcript was found in muscle (Fig. 4A,B). Fig. 4C showed that the expression level of PmAP- $2 \beta$ in WSSV-infected shrimp was higher than that in unchallenged shrimp, especially at 6 and $36 \mathrm{hpi}$, at which PmAP-2 $\beta$ was highly up-regulated for 4 -fold.

Silencing of PmAP-2 $\beta$ lowered WSSV infection. An appropriate amount of PmAP-2 $\beta$ dsRNA to be used in gene silencing experiments was examined in $P$. monodon. Shrimp were doubly injected with $10 \mu \mathrm{g} P \mathrm{mAP}-2 \beta$ dsRNA per $1 \mathrm{~g}$ shrimp and either 5 or $10 \mu \mathrm{g} P m \mathrm{AP}-2 \beta$ dsRNA per $1 \mathrm{~g}$ shrimp at $24 \mathrm{~h}$ interval. Hemocytes were collected at $24 \mathrm{~h}$ after second injection and 10, $5 \mu \mathrm{g}$ PmAP-2 $\beta$ dsRNA injection seemed to show stronger effect on silencing PmAP-2 $\beta$ expression than 10, $10 \mu \mathrm{g} P m$ AP- $2 \beta$ dsRNA injection (Fig. 5A). As a result, 10, $5 \mu \mathrm{g} P m \mathrm{AP}-2 \beta$ dsRNA injection was used in RNAi experiments. As shown in Fig. 5B, PmAP-2 $\beta$ was significantly up-regulated 
A)



B)

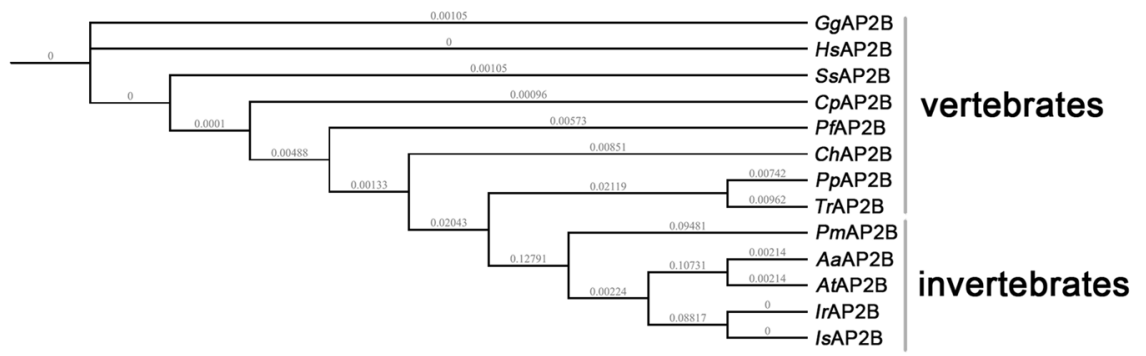

Figure 2. Analysis of nucleotide and amino acid sequences of PmAP-2 $\beta$. (A) Nucleotide and amino acid sequence of PmAP-2 $\beta$. Start codon is in a box and polyadenylation signal is underlined. Secondary structure prediction was carried out using Jpred $4^{61}$. (B) Phylogenetic tree of AP-2 $\beta$ from invertebrates and vertebrates. Sequences of AP-2 $\beta$ were obtained from The National Center for Biotechnology Information (NCBI) and phylogenetic tree was generated by iTOL. AP-2ßs are from Gorilla gorilla gorilla (GgAP2B, G3RHU1); Homo sapiens (HsAP2B, P63010); Sus scrofa (SsAP2B,acc. NO.NP_001230112.1); Cavia porcellus (CpAP2B, acc. NO.XP_003469646.1); Patagioenas fasciata monilis (PfAP2B, acc.NO. OPJ78715.1); Crotalus horridus (ChAP2B, acc. NO.GAAZ01000182.1); Poeciliopsis prolifica (PpAP2B, acc. NO.GBYX01461436.1); Takifugu rubripes (TrAP2B, acc. NO.XP_003970865.1); Penaeus monodon (PmAP2B); Anopheles albimanus (AaAP2B, acc. NO.A0A182FRP2); Anopheles triannulatus (AtAP2B, acc. NO.GGFK01000869.1); Ir, Ixodes Ricinus (acc. NO.GEFM01002526.1); Is, Ixodes scapularis (acc. NO. XP_002412040.1). 


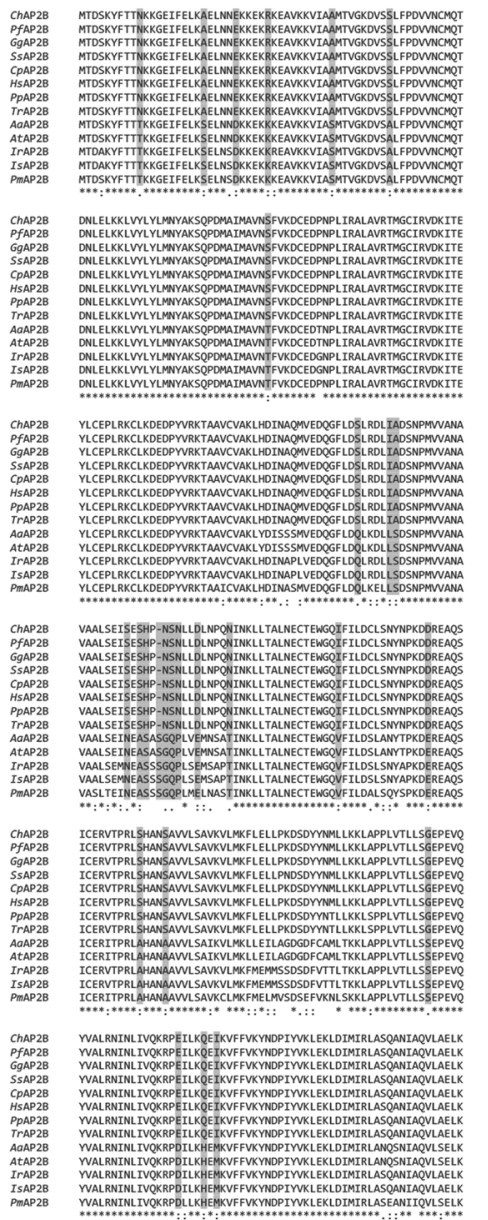

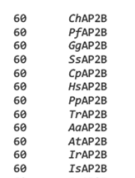


768
768
768
768
768
768
770
770
764
764
769
769
764

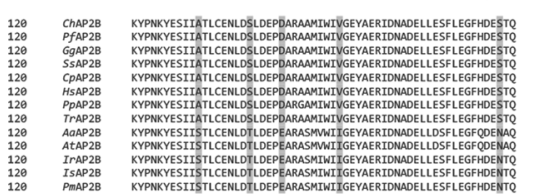

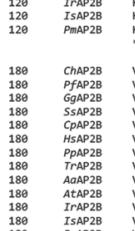
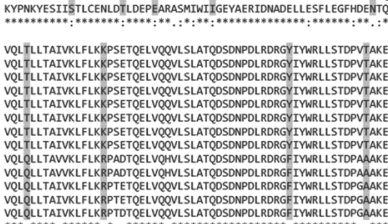

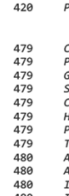
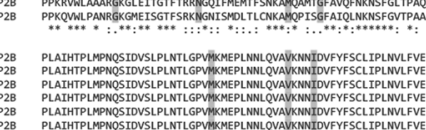

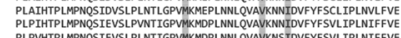

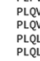

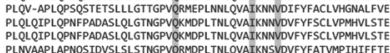
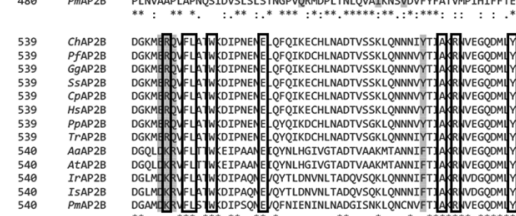

828
828
8828
828
828
838
838
832
823
823
829
824

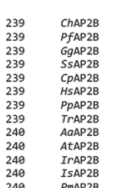
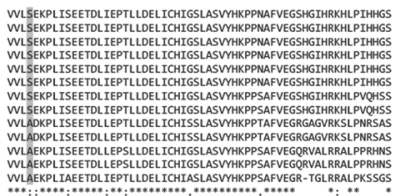

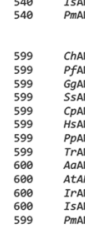

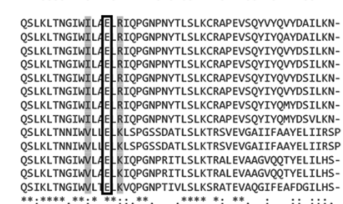

388
3888
888
888
888
889
890
383
883
889
889
884
8

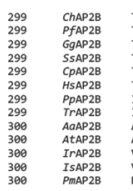

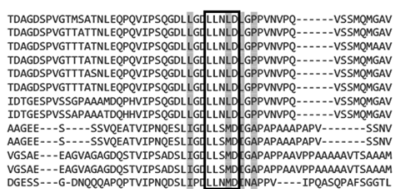

653
653
653
653
653
653
653
653
645
645
655
655
651

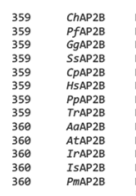

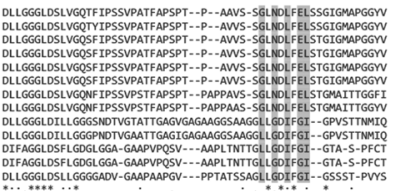

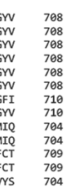

Figure 3. Multiple sequence alignment of AP- $2 \beta$ from invertebrates and vertebrates. AP- $2 \beta$ s are from Crotalus horridus (ChAP2B, acc. NO.GAAZ01000182.1); Patagioenas fasciata monilis (PfAP2B, acc.NO. OPJ78715.1); Gorilla gorilla gorilla (GgAP2B, G3RHU1); Sus scrofa (SsAP2B, acc. NO.NP_001230112.1); Cavia porcellus (CpAP2B, acc. NO.XP_003469646.1); Homo sapiens (HsAP2B, P63010); Poeciliopsis prolifica (PpAP2B, acc. NO.GBYX01461436.1); Takifugu rubripes (TrAP2B, acc. NO.XP_003970865.1); Anopheles albimanus (AaAP2B, acc. NO.A0A182FRP2); Anopheles triannulatus (AtAP2B, acc. NO.GGFK01000869.1); Ir, Ixodes Ricinus (acc. NO.GEFM01002526.1); Is, Ixodes scapularis (acc. NO. XP_002412040.1); Penaeus monodon (PmAP2B).

during WSSV infection and shrimp injected with GFP dsRNA possessed similar level of PmAP-2 $\beta$ transcript to that of normal shrimp challenged by WSSV. Meanwhile, shrimp repeatedly injected with $10,5 \mu \mathrm{g} P \mathrm{mAP}-2 \beta$ dsRNA and WSSV showed dramatically lower level of PmAP- $2 \beta$ transcripts at 6, 12, 24, 36 and 48 hpi, in comparison with those in WSSV-challenged and WSSV-challenged + GFP knockdown shrimp. This result indicated that PmAP- $2 \beta$ was specifically knockdown throughout the experiments. PmAP- $2 \beta$ silenced shrimp exhibited a significantly lower level of WSSV IE-1 transcripts at 24 and $36 \mathrm{hpi}$, compared with those in WSSV-challenged and WSSV-challenged + GFP knockdown shrimp (Fig. 5C). In addition, PmAP- $2 \beta$ silenced shrimp had a significant lower viral copy numbers at 12, 24 and 36 hpi than that of WSSV-challenged and WSSV-challenged + GFP knockdown shrimp (Fig. 5D). These suggested that PmAP-2 $\beta$ silencing affected WSSV propagation. Similar conclusions were drawn from mortality assay, in which delayed mortality of shrimp was observed in PmAP- $2 \beta$ knockdown group (Fig. 5E). Clearly, PmAP-2 $\beta$ is involved in WSSV infection.

PmAP-2 $\beta$ localized with WSSV during viral infection. In unchallenged hemocytes, immuno-gold particles, representing PmAP-2 $\beta$, were presented in a wide area of cytoplasm (red arrows) (Fig. 6A). On the contrary, PmAP-2 $\beta$ was mostly accumulated at the plasma membrane of WSSV-challenged shrimp hemocytes and the internalized WSSV particles were surrounded by PmAP-2 $\beta$ proteins (black arrows) (Fig. 6B). TEM visualization of PmAP-2 $\beta$ and viral particles confirmed that WSSV enters shrimp cells via clathrin-mediated endocytosis.

Effects of PmAP-2 $\beta$ knockdowns on signaling pathways and immune responses during WSSV infection. WSSV entry may trigger several signaling pathways, including Toll, Imd and JAK/STAT pathways, as well as other immune related genes such as antimicrobial peptides. In this work, the gene expression of PmDOME, $P m S$ TAT, $P m$ Spätzle, $P m$ Dorsal, $P m$ Relish and ALFP $m 3$ were determined by qRT-PCR. $P m$ DOME and PmSTAT present in the JAK/STAT signaling pathway, while PmSpätzle and PmDorsal are in the Toll pathway 
A)
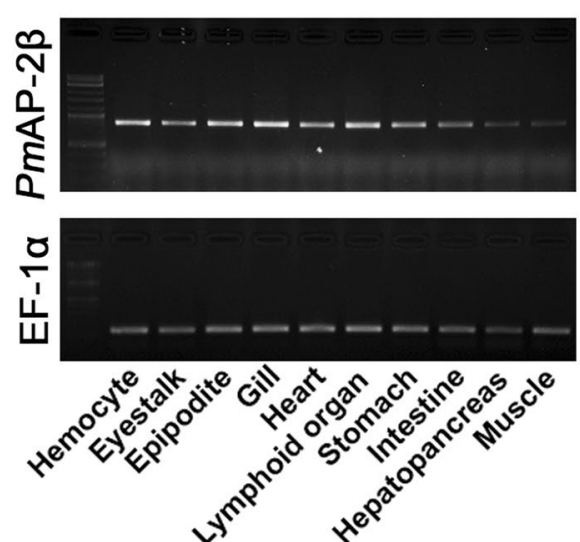

B)

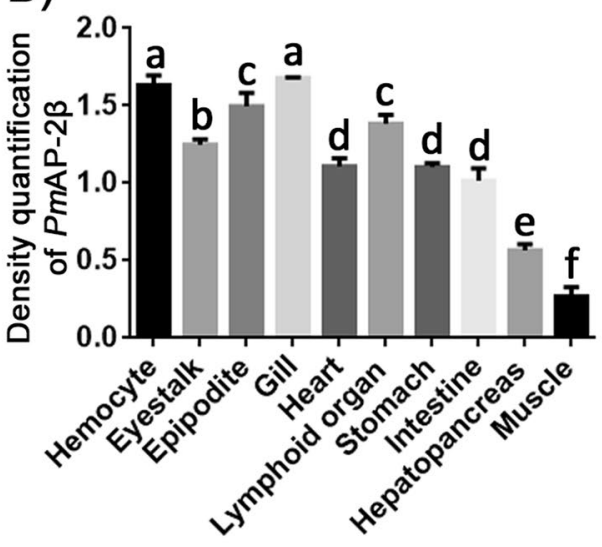

C)

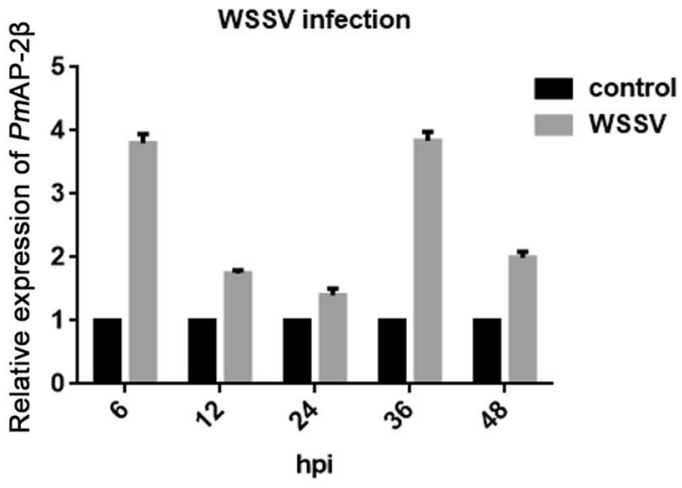

Figure 4. Analysis of PmAP-2 $\beta$ expression in shrimp. (A) Tissue distribution of PmAP-2 $\beta$ in hemocyte, eyestalk, epipodite, gill, heart, lymphoid organ, stomach, intestine, hepatopancreas and muscle. EF-1 $\alpha$ was used as an internal control. (B) RT-PCR was used to determine PmAP- $2 \beta$ gene expression in various shrimp tissues. The different letters above the bars represent significant differences at $p<0.05$. (C) Relative expression ratio of PmAP-2 $\beta$ gene in WSSV infected shrimp, analyzed by quantitative Real-time RT-PCR.

and PmRelish belongs to the Imd pathway. ALFPm3 was reported to reduce WSSV propagation ${ }^{36}$ and interact with several WSSV structural proteins ${ }^{37}$. During WSSV infection, levels of PmDOME and PmSTAT transcripts in PmAP-2 $\beta$ silenced shrimp were significantly higher than that in normal shrimp infected with WSSV (Fig. 7A,B). Especially at $12 \mathrm{hpi}, P m D O M E$ and PmSTAT were up-regulated by 4.3 -fold and 25.7 -fold, respectively. PmAP-2 $\beta$ silencing increased expression of PmSpätzle at 6,12, 24 hpi and PmDorsal transcription level at 12 and $36 \mathrm{~h}$ post-WSSV infection (Fig. 7C,D). The expression of PmRelish was highest at 36 hpi and increased by 2.1 -fold, in comparison to uninfected shrimp (Fig. 7E). The PmRelish transcript levels of WSSV-challenged PmAP-2 $\beta$ knockdown shrimp were increased at 12 and $48 \mathrm{hpi}$, but significantly decreased at 24 and $36 \mathrm{hpi}$, when compared with normal shrimp infected with WSSV. Overall, the data suggested that PmAP- $2 \beta$ may be related to the JAK/ STAT and the Toll pathways, but not the Imd pathway. During WSSV infection, ALFPm3 was up-regulated by 5.9-, 4.4- and 3.3-fold at 6, 12 and 24 hpi (Fig. 7F). Surprisingly, expression of ALFPm3 was significantly enhanced in PmAP-2 $\beta$ silenced shrimp by 13-, 20- and 7.2-fold at 6, 12 and $24 \mathrm{hpi}$, respectively.

\section{Discussion}

This work aims to characterize the clathrin adaptor proteins from P. monodon, PmAP-2 $\beta$ and PmAP- $2 \mu$ and study clathrin-mediated endocytosis during WSSV infection. Previously, Pm-clathrin coat AP17 or PmAP-2 $\sigma$ has been characterized ${ }^{22}$. Immunofluorescence confocal microscopy showed that level of Pm-clathrin coat AP17 was increased during WSSV infection and Pm-clathrin coat AP17 co-localized with WSV477, a Cys2/Cys2-type zinc finger regulatory protein with ATP/GTP-binding activity, in the cytoplasm of shrimp hemocytes (Fig. 1A). It is likely that Pm-clathrin coat AP17 was up-regulated in response to WSSV infection. In a previous report, silencing WSV477 resulted in a reduction of viral late gene VP28 transcript. In addition, WSV477 was reported to bind to a Kazal serine proteinase inhibitor SPIPm $2^{38}$.

$P m$-clathrin coat AP17 gene silencing and CPZ treatment resulted in a lower expression of WSSV-IE1 (Fig. 1B). This indicated that clathrin-mediated endocytosis is involved in WSSV infection in P. monodon. CPZ treatment showed stronger inhibitory effects on WSSV infection than that of $P m$-clathrin coat AP17 gene knockdown. Pm-clathrin coat AP17 gene silencing interferes with the assembly of clathrin and the formation of coated pits on the plasma membrane, while CPZ inhibits clathrin coated vesicles from recycling. 
A)

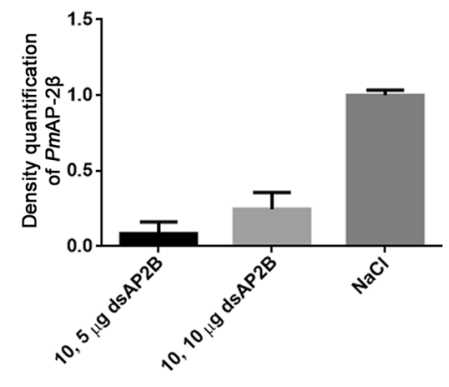

C)

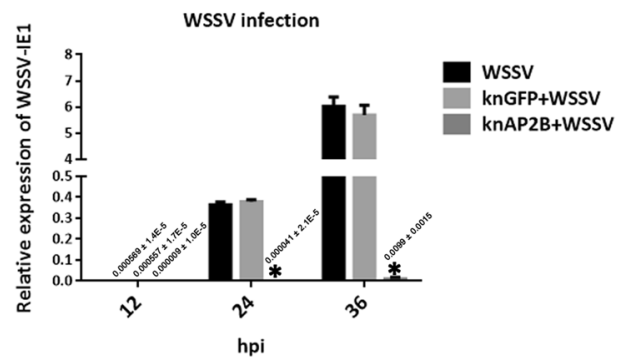

E)

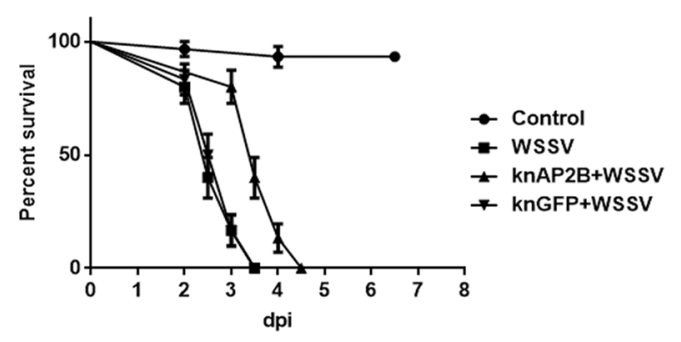

B)

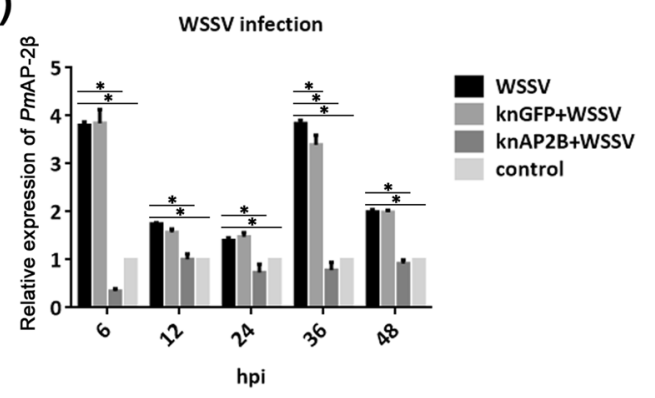

D)

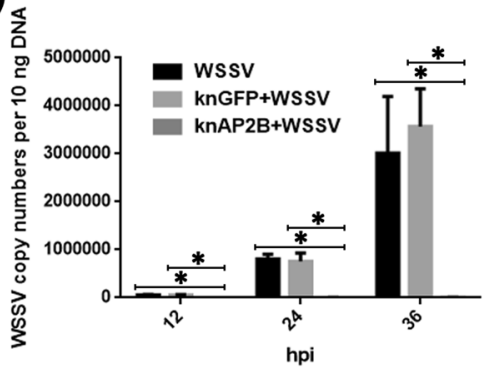

Figure 5. Effect of PmAP-2 $\beta$ knockdown during WSSV infection. (A) Determination of PmAP-2 $\beta$ transcript level in P. monodon hemocytes after RNAi-mediated gene silencing. Shrimp were double injected with either 10 and $5 \mu \mathrm{g}$ PmAP-2 $\beta$ dsRNA per $1 \mathrm{~g}$ of shrimp or 10 and $5 \mu \mathrm{g}$ PmAP- $2 \beta$ dsRNA per $1 \mathrm{~g}$ of shrimp or NaCl. The PmAP-2 $\beta$ transcript level was measured by Quantitative Real-time RT-PCR and normalized to that of EF1- $\alpha$ gene (an internal control). (B) Expression level of PmAP-2 $\beta$ in unchallenged, WSSV-challenged, WSSVchallenged + GFP knockdown and WSSV-challenged + PmAP- $2 \beta$ knockdown shrimp at 6, 12, 24, 36, 48 hpi. Quantitative Real-time RT-PCR was performed to analyze PmAP- $2 \beta$ transcript levels. (C) Determination of WSSV IE1 transcript level in WSSV-challenged, WSSV-challenged + GFP knockdown and WSSVchallenged $+P m$ AP- $2 \beta$ knockdown shrimp at 12, 24 and 36 hpi. The mRNA expression levels of WSSV IE1 were determined by Quantitative Real-time RT-PCR. (D) Analysis of WSSV copy number in WSSV-challenged, WSSV-challenged + GFP knockdown and WSSV-challenged + PmAP-2 $\beta$ knockdown shrimp at 12, 24 and 36 hpi. WSSV copy number was determined as described in Jaturontakul et al. ${ }^{54}$. (E) Cumulative mortality of shrimp after challenged with WSSV. Shrimp were divided into 4 groups (control, WSSV-challenged, WSSVchallenged + GFP knockdown and WSSV-challenged $+P m A P-2 \beta$ silenced shrimp) with 12 individuals per group. The data are shown as the mean \pm standard deviation. An asterisk indicates significant differences from control group $(p<0.05)$. All experiments were carried out in triplicates.

The complete PmAP- $2 \beta$ and PmAP- $2 \mu$ cDNA sequences were reported and the amino acid sequences were analyzed. AP- $2 \beta$ and AP- $2 \mu$ are highly conserved across diverse phyla. In addition, the recombinant PmAP- $2 \beta$ and PmAP- $2 \mu$ were successfully expressed and purified as shown in Fig. S1 in Supplementary Information. An apparent molecular weight of PmAP- $2 \beta$ and PmAP- $2 \mu$ determined by SDS-PAGE was in a good agreement with the calculated molecular mass.

PmAP-2 $\beta$ was up-regulated in response to WSSV infection (Fig. 4C). At 6 and 36 hpi, the PmAP-2 $\beta$ transcript levels in WSSV-infected shrimp were increased by 4 -fold, compared with uninfected shrimp. PmAP-2 $\beta$ silenced shrimp showed significantly lower WSSV-IE1 mRNA level than that in normal shrimp infected with WSSV (Fig. 5C). It was previously shown that AP-2 is important for endocytic coated-pit and coated-vesicle formation at the plasma membrane and very few clathrin coats were observed after AP-2 knockdown in BSC1 and HeLa cells ${ }^{39}$. Disruption of clathrin-dependent trafficking by PmAP- $2 \beta$ silencing resulted in a reduction of WSSV copy number (Fig. 5D). This was in agreement with the cumulative mortality experiment showing that $P m A P-2 \beta$ silencing decreased mortality rates (Fig. 5E). TEM demonstrated that $P m A P-2 \beta$ accumulated at plasma 
A)

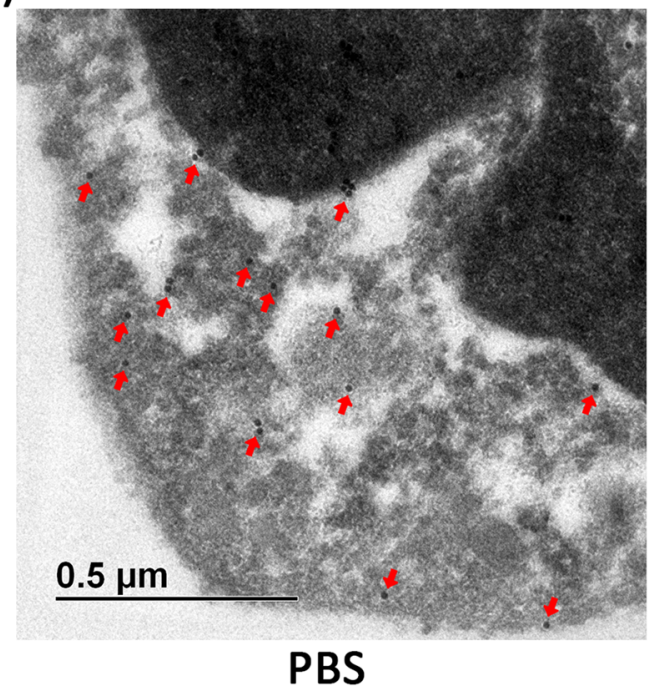

B)

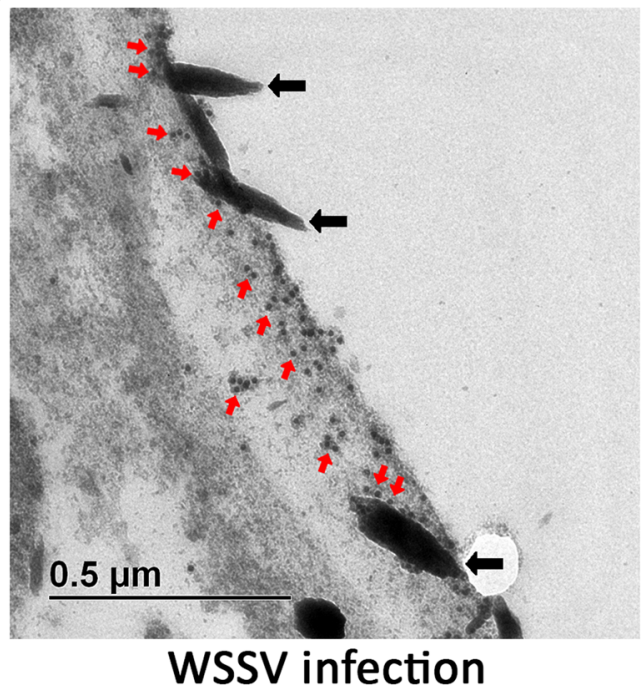

Figure 6. Localization of PmAP-2 $\beta$ in uninfected (A) and WSSV infected (B) hemocyte cells by immunoelectron microscopy. PmAP- $2 \beta$ was detected using $10 \mathrm{~nm}$ gold-conjugated anti-AP-2 $\beta$ antibody. Red arrows indicate gold particles and black arrows show WSSV particles.

membrane and facilitated WSSV entry (Fig. 6B). To our knowledge, this is the first report showing clear vision of WSSV internalized into the host cells via clathrin-dependent endocytosis.

Our results confirmed that WSSV internalizes via clathrin-mediated endocytosis. Similar findings were reported in crayfish hematopoietic tissues ${ }^{13,14}$ and the pacific white shrimp L. vannamei ${ }^{12}$. Huang, Z. et al. 2013 suggested that WSSV use the caveolae-mediated endocytosis to enter the shrimp cells ${ }^{40}$. Chen, R. et al. 2016 showed that WSSV entry into hematopoietic tissues of the crayfish is dependent on multiple endocytic routes, including clathrin-mediated endocytosis, macropinocytosis and caveolae-dependent endocytosis ${ }^{14}$. PmAP- $2 \beta$ knockdown resulted in a delayed mortality, but did not completely inhibit WSSV infection. This suggested that WSSV may enter shrimp cells via multiple routes.

Relative transcription levels of immune related genes in PmAP- $2 \beta$ silenced shrimp were compared with those in normal shrimp during WSSV infection (Fig. 7). PmAP-2 $\beta$ silencing resulted in up-regulation of PmDOME, $P m S T A T, P m$ Spätzle and $P m$ Dorsal during WSSV infection. It is possible that PmAP-2 $\beta$ is somehow associated with the JAK/STAT and the Toll pathway. In contrast, effect of PmAP- $2 \beta$ knockdown on PmRelish expression level was less evident, suggesting that PmAP-2 $\beta$ may not involve in the Imd pathway.

ALFPm 3 was up-regulated in response to WSSV infection. PmAP- $2 \beta$ silencing enhanced ALFPm 3 expression at the highest level by 20 -fold at $12 \mathrm{~h}$ after WSSV challenge (Fig. 7F). It was shown previously that ALFPm 3 exhibited anti-WSSV activity via binding to the viral structural proteins, resulting in diminishing of WSSV virions ${ }^{37,41}$. A significant increase of ALFPm3 expression may cause a delayed mortality (Fig. 5E).

Transcriptomic and comparative proteomic data have shown that shrimp Toll receptors play a role during WSSV infection ${ }^{42,43}$. An RNAi study of a Toll receptor from giant freshwater prawn Macrobrachium rosenbergii (MrToll) showed that the ALF genes (ALF2, ALF3, ALF4 and ALF5) were regulated by MrToll during WSSV challenge $^{44}$. Li, H. et al., 2018 reported that Toll4 from L. vannamei inhibited WSSV infection through activation of Dorsal to induce antimicrobial peptides, including ALF and lysozyme ${ }^{45}$. Injection of PmSpätzle 1 into a normal shrimp increased ALFPm3, crustinPm1, crustinPm 7 and penaeidin 3 synthesis ${ }^{46}$. Based on these evidences, it is possible that $P m A P-2 \beta$ silencing may activate the Toll pathway through P $m$ Dorsal, and possibly PmSpätzle, leading to a significant increase of ALFPm3 transcription during WSSV infection (Fig. 7).

In Drosophila, recruitment and trafficking of the clathrin-AP complexes into endocytic vesicles towards the lysosome is required to activate JAK/STAT signaling ${ }^{47}$. In L. vannamei, silencing STAT decreased shrimp mortality caused by WSSV and specific inhibitor of STAT3 (S3I-201) inhibited WSSV replication in hematopoietic tissue of crayfish C. quadricarinatus ${ }^{48}$. These findings seemed to contradict our results, whereby PmAP-2 $\beta$ silencing, which may interrupt clathrin-vesicle formation and trafficking, resulted in activation of $P m D O M E$ and $P m S T A T$ in the JAK/STAT pathway (Fig. 7A,B). During WSSV infection, PmSTAT in PmAP-2 $\beta$ silenced shrimp was up-regulated by 25.7 -fold at $12 \mathrm{hpi}$ (Fig. 7B). PmAP-2 $\beta$ knockdown shrimp also had a reduced mortality rate caused by WSSV (Fig. 5E). Based on our results, we hypothesized that PmAP-2 $\beta$ silencing may trigger the JAK/ STAT pathway, leading to anti-WSSV response. $L v$ JAK silencing caused higher mortality ${ }^{49}$ and knockdown of LvSOC2, a negative feedback loop regulator of JAK/STAT pathway in L. vannamei, resulted in less WSSV infection $^{50}$. More recently, Wang, W. et al., 2019 reported that inhibition of the JAK/STAT pathway in Drosophila by WSV181 could enhance WSSV replication ${ }^{51}$. These findings indicated that the JAK/STAT pathway could function as anti-viral response. Role of clathrin-AP2 on the JAK/STAT pathway need to be further investigated.

When clathrin-dependent endocytosis is interrupted, WSSV may enter shrimp cells via alternative routes e.g. caveolae-dependent endocytosis and macropinocytosis ${ }^{14}$. Caveolae are cholesterol- and sphingolipid-rich 
A)
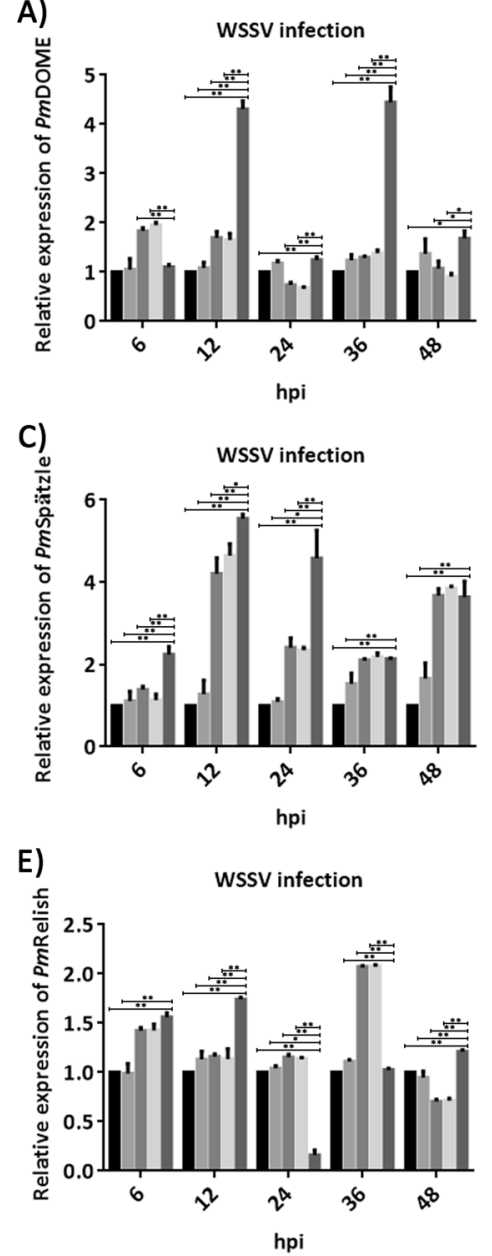

B)

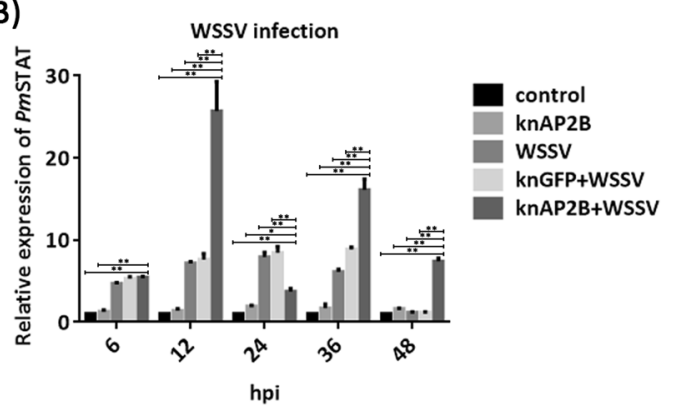

D)

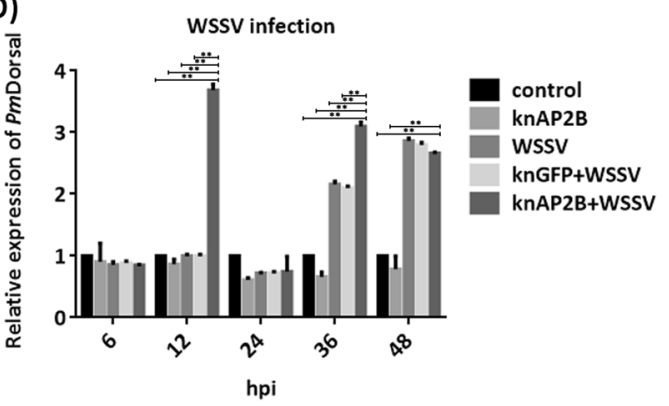

F)

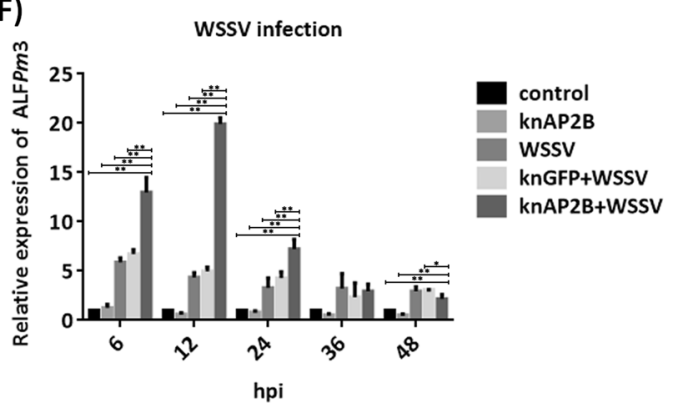

Figure 7. Relative transcription levels of immune related genes in PmAP- $2 \beta$ silenced shirmp during WSSV infection. Shrimp hemocytes were collected for qRT-PCR analysis of PmDOME (A), PmSTAT (B), PmSpätzle (C), PmDorsal (D), PmRelish (E) and ALFPm3 transcript levels at 6, 12, 24, 36 and 48 hpi. The immune relate genes expression in each groups were calculated relative to that of the control. Data are presented as mean \pm standard deviations. An asterisk $(*)$ and double-asterisk $(* *)$ represents significant differences from control group $(p<0.05)$ and $(p<0.01)$, respectively.

smooth invaginations of the plasma membrane that mediate internalization of various molecules. Caveolae are referred as clathrin-independent endocytosis and contain caveolins as the main structural proteins ${ }^{52}$. Caveolin-1 was reported to inhibit STAT5a activation by direct interaction with JAK2 ${ }^{53}$. Caveolin proteins possess the caveolin-scaffolding domain, which is similar to the SOCS kinase inhibitory region (KIR) domain, so caveolin might function as negative regulators of receptor signaling pathway via JAK/STAT.

In conclusion, this work has confirmed that WSSV enter shrimp cells via clathrin-mediated endocytosis. The complete sequences of a full-length cDNA for PmAP- $2 \beta$ and PmAP- $2 \mu$ were reported and the recombinant proteins were expressed and purified. Silencing of clathrin AP-2 components, endocytosis inhibitor, immunofluorescence confocal microscopy and TEM suggested that clathrin-mediated endocytosis play an important role during WSSV infection. In addition, PmAP- $2 \beta$ may be related to the JAK/STAT and the Toll pathways in response to WSSV.

\section{Methods}

Shrimp. Pathogen-free black tiger shrimp, P. monodon, of about $3.5 \pm 0.1 \mathrm{~g}$ bodyweight were obtained from Charoen Pokphand Farm in Chanthaburi Province, Thailand. The animals were kept in aerated water within $120 \mathrm{~L}$ laboratory tanks at temperature of $28 \pm 4^{\circ} \mathrm{C}$ and a salinity of $20 \mathrm{ppt}$ for at least 1 week prior to the experiments.

Preparation of WSSV stock. WSSV was prepared from the gill tissue of WSSV-infected moribund shrimp using centrifugation and membrane filtration as described in Jaturontakul et al. ${ }^{54}$.

Total RNA isolation and first-stranded cDNA synthesis. Shrimp tissues were collected and homogenized in FARB buffer (Tissue Total RNA mini kit, Favorgen, Taiwan). Total RNA was extracted and the first-strand 
cDNA was synthesised by First-strand cDNA Synthesis Kit (Thermo Fisher, USA). The cDNA was kept at $-20^{\circ} \mathrm{C}$ until further experiments.

Effect of clathrin coat AP17 gene silencing and chlorpromazine (CPZ) pre-treatment on WSSV propagation in shrimp. P. monodon were divided into four groups and each group contained nine shrimp. In group 1 , shrimp were injected with $150 \mathrm{mM} \mathrm{NaCl}$, while in group 2 and 3 , shrimp were injected with $10 \mu \mathrm{g}$ of GFP dsRNA per $1 \mathrm{~g}$ of shrimp and $10 \mu \mathrm{g}$ of clathrin coat AP17 dsRNA per $1 \mathrm{~g}$ of shrimp, respectively. Shrimp in group 4 were given $0.25 \mu \mathrm{g} \mathrm{CPZ}$ per $1 \mathrm{~g}$ of shrimp. After $24 \mathrm{~h}$, WSSV $\left(\sim 6 \times 10^{6}\right.$ viral copies $)$ were mixed with either $150 \mathrm{mM} \mathrm{NaCl}, 10 \mu \mathrm{g}$ of GFP dsRNA per $1 \mathrm{~g}$ of shrimp or $10 \mu \mathrm{g}$ of $P m$-clathrin coat AP17 dsRNA per $1 \mathrm{~g}$ of shrimp and injected into shrimp. At $12 \mathrm{~h}$ post injection, hemolymph was collected for total RNA extraction and cDNA synthesis. To determine the intermediate early gene of WSSV IE- 1 transcript levels, quantitative Real-time RT-PCR was carried out as $95^{\circ} \mathrm{C}$ for $30 \mathrm{~s}, 40$ cycles of $95^{\circ} \mathrm{C}$ for $5 \mathrm{~s}$ and $55^{\circ} \mathrm{C}$ for $10 \mathrm{~s}$, using specific primers for WSSV-IE1 (See Supplementary Information, Table S1). Elongation factor-1 alpha (EF-1 $\alpha$ ) gene was used as an internal control. The experiment was performed in triplicates. Mathematical model was used to analyze the threshold cycle $\left(\mathrm{C}_{\mathrm{T}}\right)^{55}$. Statistical analysis was performed using the one-way ANOVA followed by post hoc test. The result differences were considered significant at $p<0.05(*), p<0.01(* *)$.

Comparative $C_{\mathrm{T}}$ method was used to compare the gene expression in two different samples. The fold change of gene expression was calculated using the following formula.

$$
\text { Fold change }=2^{-\Delta \Delta \mathrm{CT}}
$$

$$
\begin{aligned}
\Delta \Delta C_{T}= & {\left[\left(C_{\mathrm{T}} \text { gene of interest }-C_{\mathrm{T}} \text { internal control }\right) \text { sample } \mathrm{A}-\left(C_{\mathrm{T}} \text { gene of interest }-C_{\mathrm{T}} \text { internal control }\right)\right.} \\
& \text { sample } \mathrm{B})]
\end{aligned}
$$

Immunofluorescence and confocal microscopy. A procedure used to detect Pm-clathrin coat AP17 by immunofluorescence and confocal microscopy was previously describe in Jatuyosporn et al. ${ }^{22}$. Either WSSV solution or $150 \mathrm{mM} \mathrm{NaCl}$ was injected into shrimp and hemolymph was collected from three individual shrimp at $12 \mathrm{~h}$ post-injection, pooled and mixed in equal volume of $4 \%$ paraformaldehyde in PBS. Hemocytes were collected by centrifugation $\left(800 \times \mathrm{g}\right.$ for $10 \mathrm{~min}$ at $\left.4{ }^{\circ} \mathrm{C}\right)$, washed 3 times with PBS and fixed on microscope slides. Hemocytes were then incubated with $0.1 \%$ Triton X-100 in PBS for $5 \mathrm{~min}$ and washed triplicate with PBS. $P m$-clathrin coat AP17 was probed using purified rabbit anti- $P m$-clathrin coat AP17 polyclonal IgG antibody ${ }^{22}$ diluted 1:50 in PBSF (PBS with 1\% (v/v) FBS), followed by a 1:500 dilution of Alexa Fluor 488 goat anti-rabbit IgG antibody (Invitrogen). WSSV was detected by polyclonal IgG antibody specific to WSV477 ${ }^{38}$, diluted 1:50 in PBSF, followed by a 1:1000 dilution of Alexa Fluor 568 goat anti-mouse IgG antibody (Invitrogen). Nuclei were stained with 1:1500 dilution of TOPRO-3 (Invitrogen) in PBS. The microscope slides containing the stained and fixed hemocytes were coated by ProLong Gold (Invitrogen) and stored in the dark at $4{ }^{\circ} \mathrm{C}$ until they were examined by a confocal fluorescence microscopy.

Gene cloning, sequence alignment and phylogenetic analysis of PmAP-2 $\beta$ and PmAP-2 $\boldsymbol{\mu}$. The complete sequence of a full length cDNA for PmAP- $2 \beta$ and the partial cDNA sequence for PmAP- $2 \mu$ were obtained from the P. monodon EST database (http://pmonodon.biotec.or.th). 5'-Rapid Amplification of the cDNA Ends (RACE) was carried out using SMARTer RACE $5^{\prime} / 3^{\prime}$ kit (Takara, USA) and specific primers as shown in Table S1. In brief, $1 \mu \mathrm{g}$ of total RNA was used to generate $5^{\prime}$-RACE cDNA libraries, of which $25 \mu 1$ were diluted by the addition of $90 \mu \mathrm{l}$ of Tricine-EDTA and $1 \mu \mathrm{l}$ of the solution was used as a template in RACE reaction. DNA fragment of PmAP- $2 \mu$ was amplified by specific RACE primer (Table S1) with the following conditions: 25 cycles of $94^{\circ} \mathrm{C}$ for $30 \mathrm{~s}, 68^{\circ} \mathrm{C}$ for $30 \mathrm{~s}$ and $72^{\circ} \mathrm{C}$ for $3 \mathrm{~min}$. The PCR product was then purified and ligated to linearized pRACE vector. Ligation mixture was transformed into Stellar competent cell. The plasmid DNA was extracted from the positive colony for sequencing (Macrogen).

The full length PmAP- $2 \beta$ and PmAP- $2 \mu$ genes were amplified from cDNA of normal P. monodon, using PCR condition as followed: $98^{\circ} \mathrm{C}$ for $3 \mathrm{~min}$ (denaturation), followed by $30 \mathrm{cycles}$ of $98^{\circ} \mathrm{C}$ for $30 \mathrm{~s}, 60^{\circ} \mathrm{C}$ for $30 \mathrm{~s}$ and $72^{\circ} \mathrm{C}$ for 90 s (for PmAP-2 $\beta$ ) or 60 s (for PmAP- $2 \mu$ ) and a final extension at $72^{\circ} \mathrm{C}$ for $2 \mathrm{~min}$. The specific primers for amplification of PmAP-2 $\beta$ and PmAP- $\mu$ were designed to contain $5^{\prime} E c o R$ I and $3^{\prime} X h o$ I restriction sites for $P m A P-2 \beta$ and $5^{\prime} N c o$ I and $3^{\prime}$ Xho I restriction sites for PmAP- $2 \mu$ (Table S1). The amplified full-length PmAP-2 $\beta$ $(\sim 2.8 \mathrm{~kb})$ and $P m \mathrm{AP}-2 \mu(\sim 1.3 \mathrm{~kb})$ DNA fragments were ligated with pET-28b $(+)$ expression vector (Novagen). The ligation mixture was then transformed into Escherichia coli TOP10 (Invitrogen). The recombinant plasmids, pET28b-PmAP-2 $\beta$ and pET28b-PmAP- $2 \mu$, were verified by sequencing.

The polyadenylation site in PmAP- $2 \beta$ and PmAP- $2 \mu$ sequences was predicted by Poly(A) Signal Miner (http:// dnafsminer.bic.nus.edu.sg/PolyA.html $)^{56}$ and the predicted molecular weight of PmAP- $2 \beta$ and PmAP- $2 \mu$ was calculated by Compute pI/MW (https://web.expasy.org/compute_pi ${ }^{57}$. Bioinformatics analysis of PmAP- $2 \beta$ and PmAP- $2 \mu$ was carried out using the basic local alignment search tool (BlastX, http://blast.ncbi.nlm.nih.gov/) ${ }^{58}$, multiple sequence alignment (Clustal Omega, https://www.ebi.ac.uk/Tools/msa/clustalo/) ${ }^{59}$ and phylogenetic tree (iTOL, https://itol.embl.de/ $/{ }^{60}$. The secondary structure and protein domain of PmAP-2 $\beta$ and PmAP- $2 \mu$ were predicted by Jpred4 (http://www.compbio.dundee.ac.uk/jpred/) ${ }^{61}$, PROSITE (http://prosite.expasy.org) ${ }^{62}$ and InterPro (https://www.ebi.ac.uk/interpro//) ${ }^{63}$.

Expression and purification of the recombinant PmAP-2 $\beta$ and PmAP- $2 \mu$. E. coli strain BL21 (DE3) CodonPlus-RIL (Stratagene) harboring either the recombinant plasmid pET28b-PmAP-2 $\beta$ or 
pET28b-PmAP-2 $\mu$ was grown in LB broth containing $34 \mu \mathrm{g} / \mathrm{ml}$ kanamycin at $37^{\circ} \mathrm{C}$ until $\mathrm{OD}_{600}$ reached 0.4 . Then, isopropyl- $\beta$-D-thio-galactoside (IPTG) with a final concentration of $1 \mathrm{mM}$ was added into the media and incubated for $4 \mathrm{~h}$ before the bacterial cells were harvested and resuspended in 1x PBS buffer, $\mathrm{pH}$ 7.4. Cells were disrupted by sonication and inclusion bodies were collected by centrifugation at $5000 \mathrm{xg}$ for $10 \mathrm{~min}$ at $4{ }^{\circ} \mathrm{C}$. A denaturing solution containing $1 \mathrm{x}$ PBS, $\mathrm{pH} 7.4,8 \mathrm{M}$ urea and $1 \%$ Triton X-100 was used to solubilized the inclusion bodies. The recombinant PmAP- $2 \beta$ and $P m A$ P- $2 \mu$ were purified by a Ni Sepharose 6 Fast Flow column (GE Healthcare) under denaturing conditions and eluted stepwise with a denaturing solution containing $300 \mathrm{mM}$ imidazole. Protein fractions were then analyzed by $10 \%$ sodium dodecyl sulphate polyacrylamide gel electrophoresis (SDS-PAGE). The purified protein was then dialyzed against 1x PBS buffer $\mathrm{pH} 7.4$, containing 4, 2 and $0 \mathrm{M}$ urea. After removing any remaining precipitant by centrifugation, the renatured protein was analyzed by Western blotting using a primary anti-His antibody (GE Healthcare). The concentration of purified recombinant proteins were determined by bicinchoninic acid (BCA) protein assay (Pierce).

Analysis of PmAP-2 $\beta$ gene expression in shrimp. Shrimp tissues, including hemocyte, eyestalk, epipodite, gill, heart, lymphoid organ, stomach, intestine, hepatopancreas and muscle, were collected from 3 normal shrimp and total RNA was extracted by Tissue Total RNA mini kit (Favorgen), followed by cDNA synthesis using the RevertAid First Strand cDNA Synthesis Kit (Thermo Fisher). The PmAP-2 $\beta$ gene expression level in each tissue was identified by RT-PCR using $1 \mu$ of cDNA template and PmAP-2 $\beta$ (RT-PCR) primers shown in Table S1. Elongation factor $1 \alpha($ EF-1 $\alpha)$ gene was used as an internal control. The PCR reaction was initiated by $94^{\circ} \mathrm{C}$ for $3 \mathrm{~min}$, followed by $30 \mathrm{cycles}$ of $94^{\circ} \mathrm{C}$ for $30 \mathrm{~s}, 55^{\circ} \mathrm{C}$ for $30 \mathrm{~s}$ and $72^{\circ} \mathrm{C}$ for $30 \mathrm{~s}$ and a final extension at $72^{\circ} \mathrm{C}$ for $10 \mathrm{~min}$. The PCR products were then analyzed by $2 \%(\mathrm{w} / \mathrm{v})$ agarose gel electrophoresis.

Expression level of PmAP-2 $\beta$ mRNA in unchallenged and WSSV challenged shrimp hemocytes. Shrimp were injected with either $\sim 6 \times 10^{6}$ viral copies of WSSV (challenged-shrimp) or $150 \mathrm{mM} \mathrm{NaCl}$ (unchallenged-shrimp); and the hemocytes were then collected from both groups ( 3 shrimp per group) at 6, 12, 24, 36 and $48 \mathrm{~h}$ post-injection using $500 \mu \mathrm{l}$ of modified Alsever solution (MAS: $27 \mathrm{mM}$ sodium citrate, $336 \mathrm{mM}$ $\mathrm{NaCl}, 115 \mathrm{mM}$ glucose, $9 \mathrm{mM}$ EDTA, $\mathrm{pH}$ 7.0), per $500 \mu \mathrm{l}$ of hemolymph. Total RNA extraction was performed, followed by cDNA synthesis as previously described. Real-time RT-PCR was carried out using an equal amount of cDNAs in iCycler iQTM Real-Time detection system and the SsoFast EvaGreen Supermix (Bio-Rad) in the following conditions: one cycle at $95^{\circ} \mathrm{C}$ for $30 \mathrm{~s}$, followed by 40 cycles of $95^{\circ} \mathrm{C}$ for $5 \mathrm{~s}$ and $55^{\circ} \mathrm{C}$ for $10 \mathrm{~s}$, using PmAP- $2 \beta$ and EF- $1 \alpha$ (real-time PCR) primers as shown in Table S1. The experiment was carried out in triplicates. Comparative $\mathrm{C}_{\mathrm{T}}$ method was used to compare the gene expression in WSSV challenged-(sample A) and unchallenged (sample B) shrimp ${ }^{64}$ and statistical analysis was performed using the one-way ANOVA followed by post hoc test (Duncan's new multiple range test).

RNAi-mediated PmAP-2 $\beta$ gene silencing and quantitative determination of PmAP-2 $\beta$ transcript levels and WSSV copy numbers. The recombinant $P m A P-2 \beta$ and GFP plasmids were used as templates to amplify PmAP-2 $\beta$ dsRNA and GFP dsRNA, respectively. As shown in Table S1, a pair of primers specific to either PmAP-2 $\beta$ or GFP were designed, one of which contained the T7 promoter sequence at $5^{\prime}$ (double-underlined). The two PCR products (PmAP-2 $\beta$ and GFP) were separately amplified by specific primer pairs with the following conditions: $94^{\circ} \mathrm{C}$ for $3 \mathrm{~min}$, followed by 40 cycles of $94^{\circ} \mathrm{C}$ for $30 \mathrm{~s}, 55^{\circ} \mathrm{C}$ for $30 \mathrm{~s}$ and $72^{\circ} \mathrm{C}$ for $30 \mathrm{~s}$ and a final extension at $72^{\circ} \mathrm{C}$ for $10 \mathrm{~min}$. The two PCR product templates were in vitro transcribed using the T7 RiboMAX System (Promega) to produce two complementary single-stranded RNAs. After that, RQ1 RNase- free DNase was added and incubated at $37^{\circ} \mathrm{C}$ for $1 \mathrm{~h}$ to remove the DNA template, followed by phenol-chloroform extraction. Double-stranded RNA was obtained by mixing equal amounts of each of the complementary single-stranded RNAs, incubated at $70{ }^{\circ} \mathrm{C}$ for $10 \mathrm{~min}$, and slowly cooled down at room temperature. PmAP- $2 \beta$ dsRNA and GFP dsRNA were analyzed by $1 \%$ agarose gel electrophoresis and concentrations of newly synthesized dsRNAs were determined by measuring absorbance at $260 \mathrm{~nm}$.

Shrimp of approximately $3 \mathrm{~g}$ body weight were divided into four groups with three shrimp per group. Shrimp in the first control group (group 1) and the second control (group 2) were injected with either $150 \mathrm{mM} \mathrm{NaCl}$ or $10 \mu \mathrm{g}$ of GFP dsRNA per $1 \mathrm{~g}$ of shrimp, respectively, while shrimp in gene knockdown groups were injected with either 5 or $10 \mu \mathrm{g}$ of PmAP-2 $\beta$ dsRNA per $1 \mathrm{~g}$ of shrimp (group 3 and 4, respectively). The injection was repeated at $24 \mathrm{~h}$ after the first injection; and hemolymph was collected from each shrimp at further $24 \mathrm{~h}$. Total RNA extraction and cDNA synthesis were carried out as previously described. RT-PCR was performed in order to evaluate the degree of gene transcript silencing using the PmAP-2 $\beta$ and EF-1 $\alpha$ primers (Table S1). The PCR product was analyzed by $2 \%(\mathrm{w} / \mathrm{v})$ agarose gel electrophoresis, and $P m A P-2 \beta$ gene expression level was reported as relative to that of EF- $1 \alpha$.

WSSV IE-1 transcript levels in WSSV-challenged, WSSV-challenged-knGFP and WSSV-challenged-PmAP-2 $\beta$ silenced shrimp were measured by quantitative Real-time RT-PCR as described above.

To determined WSSV copy numbers in WSSV-challenged, WSSV-challenged-knGFP and WSSV-challenged-PmAP- $2 \beta$ silenced shrimp, the total DNA was extracted from shrimp hemocytes and Real-time PCR was performed as described in Jaturontakul et al. ${ }^{54}$.

Mortality assay of PmAP-2 $\beta$ silencing shrimp upon WSSV infection. $\quad$ P. monodon were separated into four groups with 12 individuals per group. Shrimp in the first control groups (group 1 and 2) and the second control group (group 3) were injected with either $150 \mathrm{mM} \mathrm{NaCl}$ (group 1 and 2) or $10 \mu \mathrm{g}$ of GFP dsRNA per $1 \mathrm{~g}$ of shrimp (group 3). Meanwhile, shrimp in gene knockdown group (group 4) were injected with $10 \mu g$ of PmAP-2 $\beta$ dsRNA per $1 \mathrm{~g}$ of shrimp. After $24 \mathrm{~h}$, WSSV $\left(\sim 6 \times 10^{6}\right.$ viral copies) were mixed with either $150 \mathrm{mM} \mathrm{NaCl}, 10 \mu \mathrm{g}$ of GFP dsRNA per $1 \mathrm{~g}$ of shrimp or $10 \mu \mathrm{g}$ of PmAP- $2 \beta$ dsRNA per $1 \mathrm{~g}$ of shrimp and injected into shrimp. The 
shrimp in control group (group 1) were injected with $150 \mathrm{mM} \mathrm{NaCl}$ instead of WSSV. The mortality was recorded every 12 hpi up to 7 days. This experiment was performed in triplicate. Data were analyzed using GraphPad Prism 6 plot, and presented as percent survival with the $p$ values calculated by logrank test.

Localization of PmAP-2 $\beta$ in unchallenged and WSSV challenged shrimp. $P$. monodon gills were dissected from unchallenged and WSSV challenged shrimp at 12 hpi. Dissected gill was fixed in $4 \%$ paraformaldehyde in PBS for $30 \mathrm{~min}$ on ice. Then, gills were washed twice by ice-cold PBS and prepared embedding with LR White Embedding Medium (EMS) according to manufacturer's protocol. The embedded tissues were cut into ultrathin section $(60-70 \mathrm{~nm})$ and placed on Formvar-supported and carbon-coated nickel grid. The primary AP- $2 \beta$ antibody (abcam) was conjugated with gold nano particles using InnovaCoat Gold Conjugation kit according to manufacturer's protocol. The conjugated antibody was diluted 1:50 in an incubation buffer (1\% BSA in PBS). The grids were incubated with blocking buffer (1\% BSA and 5\% normal Serum in PBS) for 30 min and then incubated with a diluted conjugated antibody at $4{ }^{\circ} \mathrm{C}$ overnight. After several washes with PBS and MilliQ water, the grids were stained with uranyl acetate solution for $5 \mathrm{~min}$, followed by Reynols lead citrate solutions for $2 \mathrm{~min}$ and observed under transmission electron microscope.

Immune related gene expression in PmAP-2 $\beta$ silenced shrimp during WSSV infection. Shrimp were divided into four control groups (group 1-4) and one experimental group (group 5). Shrimp in group 1 and 2 were injected with $150 \mathrm{mM} \mathrm{NaCl}$, while shrimp in group 3 and group 4 were injected with either $10 \mu \mathrm{g}$ of PmAP-2 $\beta$ dsRNA per $1 \mathrm{~g}$ of shrimp or $10 \mu \mathrm{g}$ of GFP dsRNA per $1 \mathrm{~g}$ of shrimp, respectively. Shrimp in group 5 were also injected with $10 \mu \mathrm{g}$ of PmAP-2 $\beta$ dsRNA per $1 \mathrm{~g}$ of shrimp. Double injection was performed at $24 \mathrm{~h}$ intervals. At $24 \mathrm{~h}$ after the second injection, WSSV $\left(\sim 6 \times 10^{6}\right.$ viral copies $)$ were mixed with either $150 \mathrm{mM} \mathrm{NaCl}, 10 \mu \mathrm{g}$ of GFP dsRNA per $1 \mathrm{~g}$ of shrimp or $10 \mu \mathrm{g}$ of PmAP- $2 \beta$ dsRNA per $1 \mathrm{~g}$ of shrimp and injected into shrimp. Shrimp in control group 1 and 3 were injected with $150 \mathrm{mM} \mathrm{NaCl}$ instead of WSSV. Hemolymph was collected from each shrimp at 6, 12, 24, 36 and 48 hpi. Total RNA extraction and cDNA synthesis were performed as described above. The qRT-PCR was carried out using specific primers of immune related genes as shown in Table $\mathrm{S} 1$ and the qRT-PCR condition was $95^{\circ} \mathrm{C}$ for $30 \mathrm{~s}$, followed by 40 cycles of $95^{\circ} \mathrm{C}$ for $5 \mathrm{~s}$ and $55^{\circ} \mathrm{C}$ for $10 \mathrm{~s}$. The experiment was carried out in triplicates. Comparative $\mathrm{C}_{\mathrm{T}}$ method was used to compare the immune related gene expression in two different samples, WSSV challenged normal-(sample A) and WSSV challenged PmAP-2 $\beta$ knockdown (sample B) shrimp. Statistical analysis was done using the one-way ANOVA followed by post hoc test (Duncan's new multiple range test). The result differences were considered significant at $p<0.05(*)$ and $p<0.01(* *)$.

\section{References}

1. Lightner, D. V. et al. Risk of spread of penaeid shrimp viruses in the Americas by the international movement of live and frozen shrimp. Rev. Sci. Tech. 16, 146-160 (1997).

2. Mayo, M. A. A summary of taxonomic changes recently approved by ICTV. Arch. Virol. 147, 1655-1663 (2002).

3. Mayo, M. A. Virus taxonomy - Houston 2002. Arch. Virol. 147, 1071-1076 (2002).

4. Chang, P., Lo, C., Wang, Y. \& Kou, G. Identification of white spot syndrome associated baculovirus target organs in the shrimp Penaeus monodon by in situ hybridization. Dis. Aquat. Org. 27, 131-139 (1996).

5. Flegel, T. W. \& Alday-Sanz, V. The crisis in Asian shrimp aquaculture: current status and future needs. J. Appl. Ichthyol. 14, 269-273 (1998).

6. Park, J. H., Lee, Y. S., Lee, S. \& Lee, Y. An infectious viral disease of penaeid shrimp newly found in Korea. Dis. Aquat. Organ. 34, 71-75 (1998)

7. Rajendran, K. V., Vijayan, K. K., Santiago, T. C. \& Krol, R. M. Experimental host range and histopathology of white spot syndrome virus (WSSV) infection in shrimp, prawns, crayfish and lobsters from India. J. Fish Dis. 22, 183-191 (1999).

8. Xie, X., Li, H., Xu, L. \& Yang, F. A simple and efficient method for purification of intact white spot syndrome virus (WSSV) viral particles. Virus Res. 108, 63-67 (2005).

9. van Hulten, M. C. et al. The white spot syndrome virus DNA genome sequence. Virology 286, 7-22 (2001).

10. Yang, F. et al. Complete genome sequence of the shrimp white spot bacilliform virus. J. Virol. 75, 11811-11820 (2001).

11. Chen, L. L. et al. Transcriptional analysis of the DNA polymerase gene of shrimp white spot syndrome virus. Virology 301, 136-147 (2002).

12. Wang, X. F., Liu, Q. H., Wu, Y. \& Huang, J. Litopenaeus vannamei clathrin coat AP17 involved in white spot syndrome virus infection. Fish. Shellfish. Immunol. 52, 309-316 (2016).

13. Huang, J., Li, F., Wu, J. \& Yang, F. White spot syndrome virus enters crayfish hematopoietic tissue cells via clathrin-mediated endocytosis. Virology 486, 35-43 (2015).

14. Chen, R. Y. et al. White spot syndrome virus entry is dependent on multiple endocytic routes and strongly facilitated by CqGABARAP in a CME-dependent manner. Sci. Rep. 6, 28694 (2016).

15. Bernardes, C., Antonio, A., Pedroso de Lima, M. C. \& Valdeira, M. L. Cholesterol affects African swine fever virus infection. Biochim. Biophys. Acta 1393, 19-25 (1998).

16. Husain, M. \& Moss, B. Role of receptor-mediated endocytosis in the formation of vaccinia virus extracellular enveloped particles. J. Virol. 79, 4080-4089 (2005).

17. Wang, S. et al. Entry of a novel marine DNA virus, Singapore grouper iridovirus, into host cells occurs via clathrin-mediated endocytosis and macropinocytosis in a pH-dependent manner. J. Virol. 88, 13047-13063 (2014).

18. Simmons, G. et al. Folate receptor alpha and caveolae are not required for Ebola virus glycoprotein-mediated viral infection. J. Virol. 77, 13433-13438 (2003).

19. Bartosch, B. \& Cosset, F. L. Cell entry of hepatitis C virus. Virology 348, 1-12 (2006).

20. Suzuki, T. et al. Sialidase activity of influenza A virus in an endocytic pathway enhances viral replication. J. Virol. 79, 11705-11715 (2005).

21. Piccini, L. E., Castilla, V. \& Damonte, E. B. Dengue-3 Virus Entry into Vero Cells: Role of Clathrin-Mediated Endocytosis in the Outcome of Infection. PLoS One 10, e0140824 (2015).

22. Jatuyosporn, T., Supungul, P., Tassanakajon, A. \& Krusong, K. The essential role of clathrin-mediated endocytosis in yellow head virus propagation in the black tiger shrimp Penaeus monodon. Dev. Comp. Immunol. 44, 100-110 (2014).

23. Park, S. Y. \& Guo, X. Adaptor protein complexes and intracellular transport. Biosci. Rep. 34 (2014).

24. Kaksonen, M. \& Roux, A. Mechanisms of clathrin-mediated endocytosis. Nat. Rev. Mol. Cell Biol. 19, 313-326 (2018).

25. Rapoport, I., Chen, Y. C., Cupers, P., Shoelson, S. E. \& Kirchhausen, T. Dileucine-based sorting signals bind to the beta chain of AP-1 at a site distinct and regulated differently from the tyrosine-based motif-binding site. EMBO J. 17, 2148-2155 (1998). 
26. Aguilar, R. C., Ohno, H., Roche, K. W. \& Bonifacino, J. S. Functional domain mapping of the clathrin-associated adaptor medium chains mu1 and mu2. J. Biol. Chem. 272, 27160-27166 (1997).

27. Traub, L. M. \& Bonifacino, J. S. Cargo recognition in clathrin-mediated endocytosis. Cold Spring Harb. Perspect. Biol. 5, a016790 (2013).

28. Collins, B. M., McCoy, A. J., Kent, H. M., Evans, P. R. \& Owen, D. J. Molecular architecture and functional model of the endocytic AP2 complex. Cell 109, 523-535 (2002).

29. Kirchhausen, T. et al. Structural and functional division into two domains of the large (100- to 115-kDa) chains of the clathrinassociated protein complex AP-2. Proc. Natl. Acad. Sci. USA 86, 2612-2616 (1989).

30. ter Haar, E., Harrison, S. C. \& Kirchhausen, T. Peptide-in-groove interactions link target proteins to the beta-propeller of clathrin. Proc. Natl. Acad. Sci. USA 97, 1096-1100 (2000).

31. Kelly, B. T. et al. Clathrin adaptors. AP2 controls clathrin polymerization with a membrane-activated switch. Science 345, 459-463 (2014).

32. Olusanya, O., Andrews, P. D., Swedlow, J. R. \& Smythe, E. Phosphorylation of threonine 156 of the $\mu 2$ subunit of the AP2 complex is essential for endocytosis in vitro and in vivo. Curr. Biol. 11, 896-900 (2001).

33. Conner, S. D. \& Schmid, S. L. Identification of an adaptor-associated kinase, AAK1, as a regulator of clathrin-mediated endocytosis. J. Cell Biol. 156, 921-929 (2002).

34. Fingerhut, A., von Figura, K. \& Honing, S. Binding of AP2 to sorting signals is modulated by AP2 phosphorylation. J. Biol. Chem. 276, 5476-5482 (2001).

35. Ricotta, D., Conner, S. D., Schmid, S. L., von Figura, K. \& Honing, S. Phosphorylation of the AP2 mu subunit by AAK1 mediates high affinity binding to membrane protein sorting signals. J. Cell Biol. 156, 791-795 (2002).

36. Tharntada, S. et al. Role of anti-lipopolysaccharide factor from the black tiger shrimp, Penaeus monodon, in protection from white spot syndrome virus infection. J. Gen. Virol. 90, 1491-1498 (2009).

37. Suraprasit, S. et al. Anti-lipopolysaccharide factor isoform 3 from Penaeus monodon (ALFPm3) exhibits antiviral activity by interacting with WSSV structural proteins. Antiviral Res. 110, 142-150 (2014).

38. Ponprateep, S., Phiwsaiya, K., Tassanakajon, A. \& Rimphanitchayakit, V. Interaction between Kazal serine proteinase inhibitor SPIPm2 and viral protein WSV477 reduces the replication of white spot syndrome virus. Fish. Shellfish. Immunol. 35, 957-964 (2013).

39. Boucrot, E., Saffarian, S., Zhang, R. \& Kirchhausen, T. Roles of AP-2 in clathrin-mediated endocytosis. PLoS One 5, e10597 (2010).

40. Huang, Z. J., Kang, S. T., Leu, J. H. \& Chen, L. L. Endocytic pathway is indicated for white spot syndrome virus (WSSV) entry in shrimp. Fish. Shellish. Immunol. 35, 707-715 (2013).

41. Methatham, T., Boonchuen, P., Jaree, P., Tassanakajon, A. \& Somboonwiwat, K. Antiviral action of the antimicrobial peptide ALFPm 3 from Penaeus monodon against white spot syndrome virus. Dev. Comp. Immunol. 69, 23-32 (2017).

42. Chai, Y. M., Yu, S. S., Zhao, X. F., Zhu, Q. \& Wang, J. X. Comparative proteomic profiles of the hepatopancreas in Fenneropenaeus chinensis response to white spot syndrome virus. Fish. Shellfish. Immunol. 29, 480-486 (2010).

43. Chen, X. et al. Transcriptome analysis of Litopenaeus vannamei in response to white spot syndrome virus infection. PLoS One 8, e73218 (2013).

44. Feng, J. et al. Toll receptor response to white spot syndrome virus challenge in giant freshwater prawns (Macrobrachium rosenbergii). Fish. Shellfish. Immunol. 57, 148-159 (2016).

45. Li, H. et al. RNAi screening identifies a new Toll from shrimp Litopenaeus vannamei that restricts WSSV infection through activating Dorsal to induce antimicrobial peptides. PLoS Pathog. 14, e1007109 (2018).

46. Boonrawd, S. et al. Characterization of PmSptzle 1 from the black tiger shrimp Peneaus monodon. Fish. Shellfish. Immunol. 65, 88-95 (2017).

47. Devergne, O., Ghiglione, C. \& Noselli, S. The endocytic control of JAK/STAT signalling in Drosophila. J. Cell Sci. 120, 3457-3464 (2007).

48. Wen, R., Li, F., Li, S. \& Xiang, J. Function of shrimp STAT during WSSV infection. Fish. Shellfish. Immunol. 38, 354-360 (2014).

49. Song, X. et al. A Janus Kinase in the JAK/STAT signaling pathway from Litopenaeus vannamei is involved in antiviral immune response. Fish. Shellfish. Immunol. 44, 662-673 (2015).

50. Wang, S. et al. Shrimp with knockdown of LvSOCS2, a negative feedback loop regulator of JAK/STAT pathway in Litopenaeus vannamei, exhibit enhanced resistance against WSSV. Dev. Comp. Immunol. 65, 289-298 (2016).

51. Wang, W., Pan, C., Huang, Z., Yuan, H. \& Chen, J. WSV181 inhibits JAK/STAT signaling and promotes viral replication in Drosophila. Dev. Comp. Immunol. 92, 20-28 (2019).

52. Mayor, S., Parton, R. G. \& Donaldson, J. G. Clathrin-independent pathways of endocytosis. Cold Spring Harb. Perspect. Biol. 6 (2014).

53. Park, D. S. et al. Caveolin-1-deficient mice show accelerated mammary gland development during pregnancy, premature lactation, and hyperactivation of the Jak-2/STAT5a signaling cascade. Mol. Biol. Cell 13, 3416-3430 (2002).

54. Jaturontakul, K. et al. Molecular Characterization of Viral Responsive Protein 15 and Its Possible Role in Nuclear Export of Virus in Black Tiger Shrimp Penaeus monodon. Sci. Rep. 7, 6523 (2017).

55. Pfaffl, M. W. A new mathematical model for relative quantification in real-time RT-PCR. Nucleic Acids Res. 29, e45 (2001).

56. Liu, H., Han, H., Li, J. \& Wong, L. An in-silico method for prediction of polyadenylation signals in human sequences. Genome informatics. International Conference on Genome Informatics 14, 84-93 (2003).

57. Gasteiger, E. et al. Protein identification and analysis tools on the ExPASy server. (Humana Press, 2005).

58. Altschul, S. F., Gish, W., Miller, W., Myers, E. W. \& Lipman, D. J. Basic local alignment search tool. J. Mol. Biol. 215, $403-410$ (1990).

59. Sievers, F. et al. Fast, scalable generation of high-quality protein multiple sequence alignments using Clustal Omega. Mol. Syst. Biol. 7, $539(2011)$.

60. Letunic, I. \& Bork, P. Interactive tree of life (iTOL) v3: an online tool for the display and annotation of phylogenetic and other trees. Nucleic Acids Res. 44, W242-245 (2016).

61. Drozdetskiy, A., Cole, C., Procter, J. \& Barton, G. J. JPred4: a protein secondary structure prediction server. Nucleic Acids Res. 43, W389-394 (2015).

62. Sigrist, C. J. et al. New and continuing developments at PROSITE. Nucleic Acids Res. 41, D344-347 (2013).

63. Hunter, S. et al. InterPro: the integrative protein signature database. Nucleic Acids Res. 37, D211-215 (2009).

64. Livak, K. J. \& Schmittgen, T. D. Analysis of relative gene expression data using real-time quantitative PCR and the 2(-Delta Delta C(T)) Method. Methods 25, 402-408 (2001).

\section{Acknowledgements}

We thank Charoen Pokphand Foods in Chanthaburi, Thailand for providing black tiger shrimp and Dr. Guandalupe Trinidad Zavala Padilla at UNAM for her help with TEM. This work is supported by Thailand Research Fund RSA6180069 and Genomics Research Network on Disease Resistance in Shrimp (IRN61W0001). Chulalongkorn University is gratefully acknowledged to support T.J. via the $100^{\text {th }}$ Anniversary Chulalongkorn University Fund for Doctoral Scholarship, the $90^{\text {th }}$ Anniversary of Chulalongkorn University Fund (Ratchadaphiseksomphot Endowment Fund), and the Overseas Research Experience Scholarship for Graduate Student from the Graduate School. Authors also acknowledge the UNAM-CIC-CIAD 2018 academic exchange program. 


\section{Author Contributions}

T.J. conducted gene cloning, protein expression, gene silencing experiments and TEM. P.L. assisted T.J. in shrimp culture, gene silencing experiments and qRT-PCR. P.S. involved in immunolocalization and RNAi experiments. R.S.-M. and A. O.-L. provided access to TEM and supervised T.J. on TEM experiment. A.T. and K.K. conceived and supervised the projects. K.K. designed the experiments, analyzed data and wrote the manuscript with help from T.J. All authors reviewed the results and approved the final version of the manuscript.

\section{Additional Information}

Supplementary information accompanies this paper at https://doi.org/10.1038/s41598-019-49852-0.

Competing Interests: The authors declare no competing interests.

Publisher's note Springer Nature remains neutral with regard to jurisdictional claims in published maps and institutional affiliations.

(c) Open Access This article is licensed under a Creative Commons Attribution 4.0 International License, which permits use, sharing, adaptation, distribution and reproduction in any medium or format, as long as you give appropriate credit to the original author(s) and the source, provide a link to the Creative Commons license, and indicate if changes were made. The images or other third party material in this article are included in the article's Creative Commons license, unless indicated otherwise in a credit line to the material. If material is not included in the article's Creative Commons license and your intended use is not permitted by statutory regulation or exceeds the permitted use, you will need to obtain permission directly from the copyright holder. To view a copy of this license, visit http://creativecommons.org/licenses/by/4.0/.

(c) The Author(s) 2019 